\title{
Acoustic Cues for Sound Source Distance and Azimuth in Rabbits, a Racquetball and a Rigid Spherical Model
}

\author{
Duck O. Kim ${ }^{1}$, Brian Bishop ${ }^{1}$, AND Shigeyuki Kuwada ${ }^{1}$ \\ ${ }^{1}$ Department of Neuroscience, University of Connecticut Health Center, Farmington, CT 06030, USA
}

Received: 3 February 2010; Accepted: 12 May 2010; Online publication: 5 June 2010

\begin{abstract}
There are numerous studies measuring the transfer functions representing signal transformation between a source and each ear canal, i.e., the head-related transfer functions (HRTFs), for various species. However, only a handful of these address the effects of sound source distance on HRTFs. This is the first study of HRTFs in the rabbit where the emphasis is on the effects of sound source distance and azimuth on HRTFs. With the rabbit placed in an anechoic chamber, we made acoustic measurements with miniature microphones placed deep in each ear canal to a sound source at different positions (10-160 $\mathrm{cm}$ distance, $\pm 150^{\circ}$ azimuth). The sound was a logarithmically swept broadband chirp. For comparisons, we also obtained the HRTFs from a racquetball and a computational model for a rigid sphere. We found that (1) the spectral shape of the HRTF in each ear changed with sound source location; (2) interaural level difference (ILD) increased with decreasing distance and with increasing frequency. Furthermore, ILDs can be substantial even at low frequencies when distance is close; and (3) interaural time difference (ITD) decreased with decreasing distance and generally increased with decreasing frequency. The observations in the rabbit were reproduced, in general, by those in the racquetball, albeit greater in magnitude in the rabbit. In the sphere model, the results were partly similar and partly different than those in the racquetball and the rabbit. These findings refute the common notions that ILD is negligible at low
\end{abstract}

Correspondence to: Shigeyuki Kuwada $\cdot$ Department of Neuroscience $\cdot$ University of Connecticut Health Center - Farmington, CT 06030, USA. Telephone: +1-860-6792343; fax: +1-860-6798766; email: shig@neuron.uchc.edu frequencies and that ITD is constant across frequency. These misconceptions became evident when distance-dependent changes were examined.

Keywords: head-related transfer function (HRTF), sound localization, acoustics, auditory distance, interaural time difference (ITD) cues, interaural level difference (ILD) cues, spectral cues

\section{INTRODUCTION}

There are numerous studies measuring the transfer functions representing signal transformation between a sound source and each ear canal, i.e., the head-related transfer functions (HRTFs), for various species. However, only a handful of these addressed the effects of sound source distance on HRTFs (Zahorik 2002; Kim et al. 2008; Kuwada et al. 2010a). Ability to recognize the distance of a sound source, be it predator or prey, is important for survival of an animal.

In this study, we measured the effects of sound source distance and azimuth on HRTFs in the rabbit. This is the first study of HRTFs in the rabbit, an animal that has prominent pinnas and is being increasingly used in auditory neurophysiology (e.g., Zheng et al. 2002; Coffey et al. 2006; Kuwada et al. 2006; Devore and Delgutte 2008; Ebert et al. 2008). We compared the rabbit HRTFs to those of a racquetball and a rigid sphere model (Duda and Martens 1998) that had diameters similar to the rabbit's interaural distance. This comparison was made in order to gain insights into how the various attributes of the HRTF are affected by components of the head and body not present in simple 
structures, i.e., racquetball and sphere model. The goal was to provide systematic information about how monaural and binaural acoustic cues associated with sound source location change with sound source distance, azimuth, and frequency. Such information could be used to generate virtual auditory space stimuli to investigate spatial hearing in the animal both behaviorally and physiologically. The virtual auditory space method presents HRTFfiltered signals through earphones. In humans, the perception created by the virtual space stimuli mimics the perception of the corresponding real sound presented in external space (Wightman and Kistler 1989; Kulkarni and Colburn 1998).

\section{METHODS}

\section{Sound source}

Measuring spatial signal transformations between a sound source and the ears is ideally achieved if the sound emanates from a point in space. When the sound source is not at a point in space, the consequences become increasingly problematic with decreasing distance. Because we used sound sources at close distances, this issue was important for our acoustic measurements.

We designed and constructed a sound source to approximate an acoustic point source. It consisted of a loudspeaker with a 3-in. diaphragm (Fostex FF85K) enclosed in an airtight, foam-filled box. The sound source displayed total harmonic distortion $<1 \%$ at the signal levels used. The diaphragm was sealed onto a custom rigid conical coupler with either a $15-\mathrm{mm}$ aperture and a 227- $\mathrm{mm}$ axis length or a $30-\mathrm{mm}$ aperture and a 177-mm axis length. The interior of the rigid cone had a Pellon cloth inner cone to serve as a cross-mode suppressor. The inner cone was separated from the inner wall of the outer cone by several felt standoffs (5-mm cubes). The tips of outer and inner cones were loosely packed with acoustic damping material (AcustaStuff).

We used the small aperture source for distances between 10 and $40 \mathrm{~cm}$ and the larger aperture source for distances between 56 and $160 \mathrm{~cm}$. Because the intensity decreases with increasing distance, we used the source with the larger aperture at distances beyond $40 \mathrm{~cm}$ in order to produce sufficient intensity at the microphones. The frequency responses of the small $(15 \mathrm{~mm})$ and large $(30 \mathrm{~mm})$ aperture sound sources were measured with a $1 / 2$-in. microphone (B\&K Type 4190) facing the aperture at a distance of $5 \mathrm{~cm}$. For both sound sources, the output was constant within $\pm 9 \mathrm{~dB}$ over a range of $0.2-20 \mathrm{kHz}$. The small aperture sound source level was on average $7.2 \mathrm{~dB}$ lower than the large aperture source.
We measured the directionality of each sound source for three frequency bands as a function of azimuth for each of several distances. The levels were relative to the level at $0^{\circ}$ azimuth at each distance and for each frequency band. Each sound source was fixed in space such that the axis of the conical coupler was in a horizontal plane. The azimuth and distance of the microphone (B\&K Type 4190) was varied relative to the tip of the sound source's conical coupler. An ideal point source exhibits equal level across all azimuths. Overall, our sound sources approximated this behavior. For both sound sources, for all azimuths and distances, the sound levels essentially were constant within $10 \mathrm{~dB}$ in the low and mid-frequency bands. In the high frequency band, the sound levels remained constant within $10 \mathrm{~dB}$ for azimuths between $\pm 90^{\circ}$ for the small aperture source, whereas the levels remained within $10 \mathrm{~dB}$ for azimuths between $\pm 50^{\circ}$ for the larger aperture source. In the actual recording situation with the rabbit in place, the angle subtended by the rabbits head was $\sim \pm 30^{\circ}$ at $10 \mathrm{~cm}$ distance and $\sim \pm 1.8^{\circ}$ at $160 \mathrm{~cm}$. So, for these azimuths, the sound level remained within $1 \mathrm{~dB}$ for all frequency bands. The sound source was positioned from $-180^{\circ}$ to $165^{\circ}$ in $15^{\circ}$ steps along the azimuth and from 10 to $160 \mathrm{~cm}$ in half doubling steps (factor of 1.4) along the distance. For the close distances, the azimuths towards the back could not be measured because the body of the sound source was an obstruction.

\section{Acoustical environment}

Measurements were made in an anechoic chamber designed so that the sound field complies with the performance specified in ISO 3745 (1977). All surfaces of the anechoic chamber were lined with fiberglass wedges designed to be anechoic between 0.11-200 kHz. The space between the wedge tips was approximately $9 \times 4 \times 4 \mathrm{~m}$, which permitted acoustic experiments to be conducted with sound sources both near and far from the animal.

\section{Dutch-belted rabbit}

We chose the rabbit because this animal has been used in neurophysiological studies (Kuwada et al. 2006; Nelson and Carney 2007; Devore and Delgutte 2008; Fitzpatrick et al. 2009). It is a good model because the frequency range of rabbit's hearing (Heffner and Masterton 1980) largely overlaps with that of human hearing. Furthermore, Carney et al. (2010) recently showed that the Dutch-belted rabbit can discriminate the distance of a sound source. 
The awake rabbit was wrapped in a body stocking and seated in a wire, padded cradle with its head restrained and positioned in the anechoic chamber. The rabbit's pinnae were held in an upright position through permanent loop earrings at the tip of the pinna fixed to a T-shaped trapeze. The placement of the sound source was accomplished with a custommade assembly. With the rabbit's head in a fixed position (approximately horizontal between bregma and lambda), the coordinates for the sound source position was relative to the origin that was defined as the point of intersection between the mid-sagittal plane and the line between the intertragal notches (see Fig. 1). The custom assembly allowed the sound source to be placed at any azimuth within distances between 10 and $160 \mathrm{~cm}$ at $0^{\circ}$ elevation from the above-defined origin.

Figure 1 illustrates the outer ear of the rabbit (panel A) with a positive cast of the deep, hidden part of the outer ear superimposed onto the visible

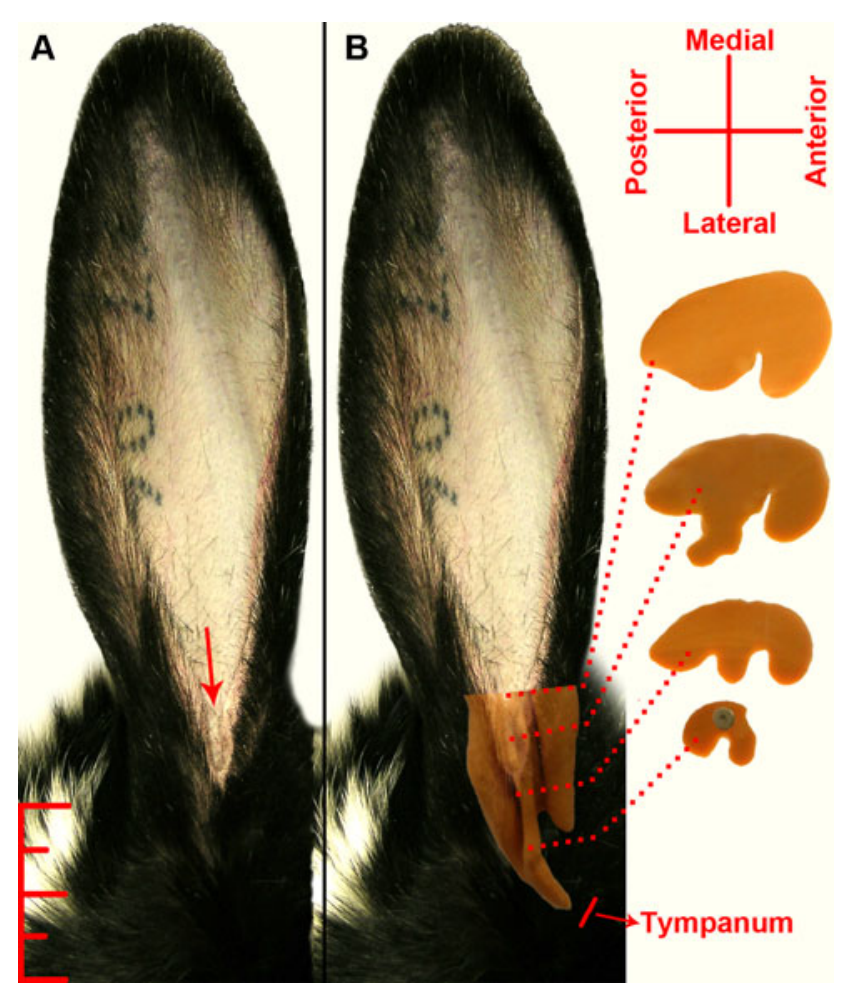

FIG. 1. A Lateral view of the right outer ear of a rabbit. The downward arrow points to a slit in the outer ear that is used as a benchmark to measure the ear length and interaural distance. B Same lateral view as in $\mathbf{A}$ with a superposed view of a cast made from dental impression material (Reprosil) to show the deeper, hidden part of the outer ear air cavity. The approximate position of the tympanum is also shown. Cross-sections of the cast at four levels are shown in the right part of panel $\mathbf{B}$. The deepest section shows an embedded miniature microphone (Knowles FG-3329, 2.5-mm diameter $\times 2.6 \mathrm{~mm}$ length) used to measure the acoustic signals in a blocked meatus approach. Scale bar is $20 \mathrm{~mm}$ divided into $5 \mathrm{~mm}$ sections. part (panel B). The visible part of the outer ear (pinna) is relatively simple and is expected to act as a collector that funnels the sound into the deeper, more complex part of the outer ear. The deeper part has ridges that create two to three air compartments (panel B) that the sound must travel through before reaching a cylindrical part that ends with the tympanum.

Cross-sections of the cast at four levels are shown in the right part of panel $\mathrm{B}$. The deepest section shows an embedded miniature microphone (Knowles FG-3329, 2.5-mm diameter $\times 2.6-\mathrm{mm}$ length) used to measure the acoustic signals. We inserted a pair of such earmold tips into the ears and then recorded ear canal signals, i.e., a blocked meatus approach (Moller et al. 1995). This location is chosen because it is near the point where the ear canal becomes cylindrical and constant in diameter. Beyond this point, it is expected that there is little, if any, influence of source direction on the acoustic signal because, in humans and cats, the signal in the ear canal was directionindependent (Wiener and Ross 1946; Wiener et al. 1966; Middlebrooks and Green 1990; Moller et al. 1995).

We used two rabbits. Rabbits 1 and 2 had body weights of 2.3 and $2.15 \mathrm{~kg}$, respectively. The lengths of the outer ear from the bottom of the slit (Fig. 5a. arrow) to the tip for rabbit 1 were 80 and $81.2 \mathrm{~mm}$ for the right and left ears, respectively. The interaural distance of this rabbit measured at the level of the slits was $57.3 \mathrm{~mm}$. Rabbit 2 died unexpectedly, and measurements of its outer ears were not available.

\section{Head-related transfer functions}

These functions were obtained by presenting a periodic logarithmic-chirp $(0.05-49 \mathrm{kHz}, 671 \mathrm{~ms}$ duration, repeated eight times) to the rabbit, at each spatial position described above. We used a PC with custom Matlab-based software interfaced to a RX6 processor (TDT). The processor output was connected to an attenuator (HP 350D) and then a power amplifier (Bryston 2B-LP) to drive the sound source. The output of each miniature microphone (Knowles FG-3329) was processed with a customdesigned amplifier-filter (40 $\mathrm{dB}$ gain, 0.03 to $30 \mathrm{kHz}$ pass band). The RX6 processor concurrently sampled, at $98 \mathrm{kHz}$, the outputs of the amplifier-filter. The frequency response of the total measurement system that included the sound source and each microphone was obtained with each microphone placed $5 \mathrm{~cm}$ in front of the sound source. This frequency response served as the reference signal for the determination of the HRTF. The frequency responses measured with the mini- 
ature microphones were indistinguishable from those measured with the $\mathrm{B} \& \mathrm{~K} 1 / 2$-in. microphone. For each sound source location, the HRTF of each ear was obtained by dividing the spectrum of the signal in the ear with the spectrum of the reference signal. The head-related impulse response (HRIR) was obtained by taking an inverse Fourier-transform of the HRTF.

In order to separate the direct signal component from late-arriving reverberant component, we applied a temporal window to the HRIR. We centered a Blackman window (Oppenheim et al. 1999) at the onset of the direct signal component. The full width of the window was $40 \mathrm{~ms}$. Thus, the effective width of the window (i.e., the width of an equivalent rectangular window) was $20 \mathrm{~ms}$. Since the HRIR was periodic where the last point was contiguous with the first point, the temporal window often encompassed both the initial portion and the last portion of the HRIR. We took a Fourier-transform of the direct HRIR to obtain the direct HRTF. The amplitude of the HRTF was converted into level in decibels (relative to the reference signal) while the phase of the HRTF was converted into "phase delay" in milliseconds (viz., phase/frequency; Blauert 1997). The amplitude and phase versus frequency functions were smoothed by convolving them with a Blackman logarithmic-frequency window with a $1 / 6$ octave half-width. This approach is analogous to that of Kulkarni and Colburn (1998). We determined ILD for each frequency by taking a difference between the monaural levels (right-left level). Likewise, we determined ITD for each frequency by taking a difference between the monaural phase delays.

\section{Racquetball}

We made measurements in a racquetball in the anechoic chamber so that we could compare it with the rabbit and the computational model of a rigid sphere. We chose a racquetball because its diameter (56 mm) is close to that of the rabbit's interaural distance. The racquetball was filled with Plaster of Paris and fitted with two miniature microphones (Knowles FG-3329) at $\pm 90^{\circ}$ azimuths on the equator. The surface of each microphone was flush with the surface of the ball.

\section{Rigid sphere model}

We used a quantitative model of a rigid sphere (Duda and Martens 1998) that incorporates sound source distance and azimuth as independent variables. We used their model without modification and derived level and phase of monaural signals. We used these signals to determine ITD and ILD as a function of frequency, distance, and azimuth as a basis for comparison with measurements in the rabbit and racquetball.

\section{RESULTS}

Level and temporal characteristics of the racquetball and rigid sphere model

We chose to make measurements in a racquetball and from a rigid sphere model and to compare these to the rabbit HRTFs. The ball and sphere model serve as simple objects of comparable size to that of the rabbit's head. Figure 2 shows the levels of the right and left microphones in the ball (A \& B) and the corresponding levels computed with the rigid sphere model (D \& E) for the sound source positioned at $90^{\circ}$ azimuth and distances between 10 and $160 \mathrm{~cm}$. In all cases, the level increased with decreasing distance and is relatively flat across frequency. Furthermore,

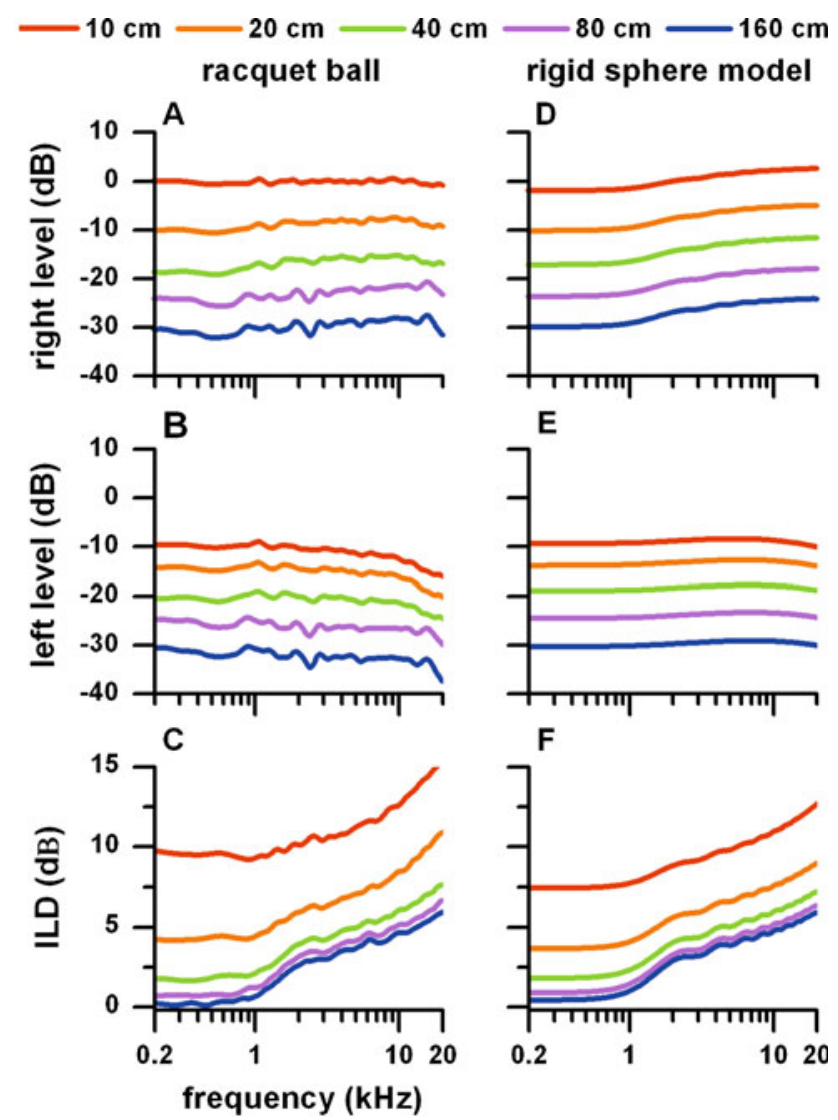

FIG. 2. Monaural levels (top two rows) and ILD (bottom row) vs. frequency measured at five distances $(10-160 \mathrm{~cm})$ and $90^{\circ}$ azimuths in a racquetball placed in an anechoic chamber (left column). The corresponding measures computed using the rigid sphere model of Duda and Martens (1998) are shown in the right column. The monaural levels were relative to a reference signal at $5 \mathrm{~cm}$ from the sound source. 
the levels measured in the ball with distance and frequency have a remarkable similarity to those computed in the model. In the bottom panels are the respective ILDs derived from the monaural levels shown above. For both the ball $(\mathrm{C})$ and sphere model (F), ILD increased with increasing frequency and decreasing distance. The measured ILDs in the ball were in remarkable agreement with the sphere model prediction except at the closest distance $(10 \mathrm{~cm})$ where the ILD in the ball was larger by $2-3 \mathrm{~dB}$ across frequency than the model.

Figure 3 shows the phase delays of the right and left microphones in the ball and the corresponding delays of the rigid sphere model for the sound source at $90^{\circ}$ azimuth (off the right microphone) and distances between 10 and $160 \mathrm{~cm}$ (ball A-E, sphere $\mathrm{G}-\mathrm{K})$. We restricted the frequency range $(0.2-2 \mathrm{kHz})$ because it is the range of stimulus frequencies where ITD sensitivity is seen in neurons in the mammalian midbrain and forebrain (Stanford et al. 1992; Fitzpatrick et al. 2000; Kuwada et al. 2006). In all cases, as expected, the delay to the left microphone (contralateral to the sound source) was longer than that to the right microphone, and the delay to both microphones increased with increasing distance. In the model, the delays were constant across frequency for all distances, whereas, in the ball, the delay decreased with decreasing frequency, and the magnitude of this decrease increased with increasing distance. In the bottom panels are the respective ITDs derived from the microphone delays shown above. For the model (panel L), ITD remained relatively constant between 0.2 and $1 \mathrm{kHz}$ for all distances and then decreased between 1 and $2 \mathrm{kHz}$ where ITDs increased slightly with decreasing distance. The ITD behavior of the ball was similar to that of the sphere model when the distance was $\geq 40 \mathrm{~cm}$. However, at close distances $(\leq 20 \mathrm{~cm})$, ITD decreased with decreasing distance and with decreasing frequency. At $0.2 \mathrm{kHz}$, the ITD decreased from $245 \mu \mathrm{s}$ at $160 \mathrm{~cm}$ to $207 \mu \mathrm{s}$ at $10 \mathrm{~cm}$, a difference of $38 \mu$ s ( $18 \%$ or $15 \%$ depending on the denominator).

\section{Variability of HRTF measurements in the rabbit}

The variability of HRTFs measured both within and across sessions was small. This was assessed by making multiple measurements at the same sound source location. We recorded ten HRTFs over 2 days in the right and left ears of a rabbit to a sound source at the same location $\left(0^{\circ}\right.$ azimuth and $20-\mathrm{cm}$ distance). In both ears, the spectral shapes remained stable across the multiple measurements. The standard deviation was less than $2.8 \mathrm{~dB}$ except at $20 \mathrm{kHz}$ where it was $5 \mathrm{~dB}$.

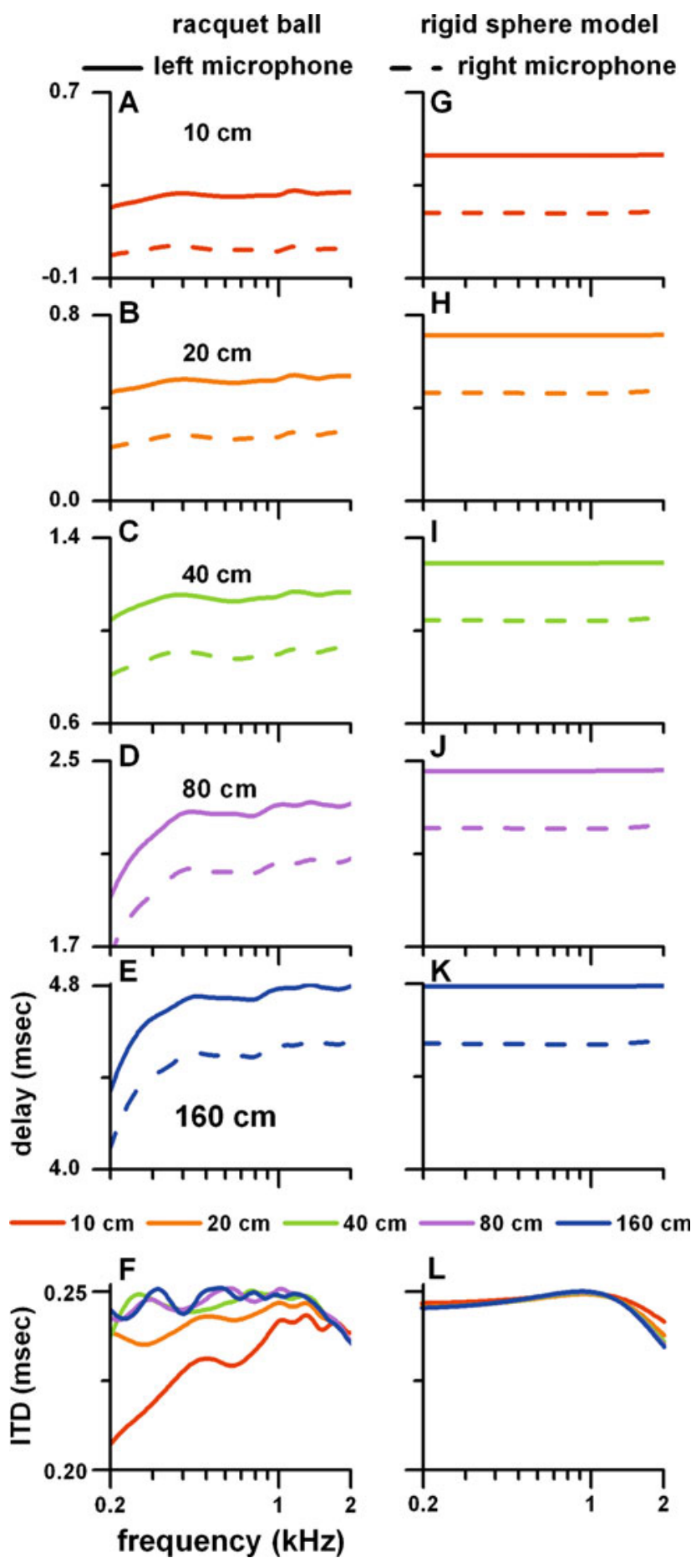

FIG. 3. Monaural delays (top five rows) and ITD (bottom row) vs. frequency measured at five distances $(10-160 \mathrm{~cm})$ and $90^{\circ}$ azimuth in a racquetball placed in an anechoic chamber (left column). The corresponding measures computed using the rigid sphere model of Duda and Martens (1998) are shown in the right column.

Monaural and binaural level characteristics of the rabbit

Comparable measurements were made in two rabbits. The HRIRs and HRTFs of one rabbit are shown in Figure 4 for different azimuths of the sound 


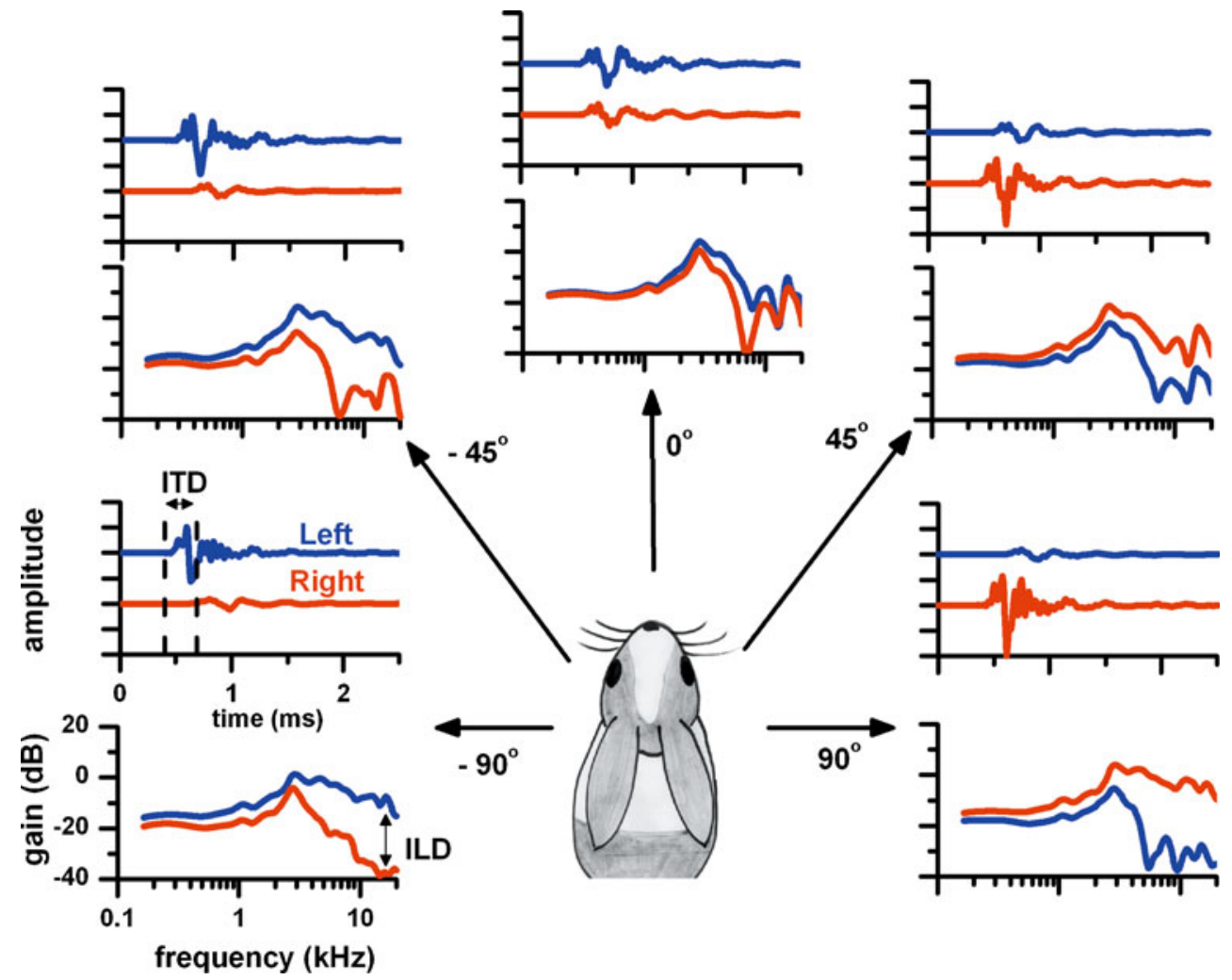

FIG. 4. HRTFs and HRIRs of a rabbit for azimuthal sound source positions of $-90^{\circ},-45^{\circ}, 0^{\circ}, 45^{\circ}$, and $90^{\circ}$ at $0^{\circ}$ elevation and distance of $40 \mathrm{~cm}$ measured in an anechoic chamber. Top panel for each position shows the impulse responses (HRIRs) and bottom panel shows the transfer functions (HRTFs) for the signals recorded at the left (blue lines) and right (red lines) ears.

source at $0^{\circ}$ elevation and at a distance of $40 \mathrm{~cm}$. Top panel for each position shows the HRIRs and bottom panel the HRTFs for the left (blue lines) and right (red lines) ears. When the sound source was facing the left ear $\left(-90^{\circ}\right)$, the amplitude of the left HRIR was noticeably larger than that of the right HRIR. This difference in the amplitudes of the HRIRs is expressed as higher levels of the left HRTF than those of the right HRTF. This is due to the head shadowing effect. The ILD generally increased with frequency (up to $\sim 40 \mathrm{~dB}$ ) because the wavelength of the sound becomes progressively smaller relative to the head width. The ITD is readily visible in the arrival times of the impulse responses of the two ears. As the sound source moved along the azimuth towards the median plane $\left(0^{\circ}\right)$, both the ILD and ITD decreased. However, even at $0^{\circ}$ azimuth, there were small differences in ILD, particularly at high frequencies. This presumably is due to slight asymmetries in the position and anatomy of the external ears. As the sound source moves towards the right ear, the relation between the two ears reverses compared with the opposite hemifield.

Figure 5 provides a detailed view of the levels of the HRTFs of the two ears (top and middle rows) of a rabbit across frequency at azimuths of $0^{\circ}, 30^{\circ}, 60^{\circ}$, and $90^{\circ}$ and distances of $10,20,40,80$, and $160 \mathrm{~cm}$. The HRTFs all show a common pattern. Between 0.2 and $1 \mathrm{kHz}$, the level remained relatively flat. Above $2 \mathrm{kHz}$, the level increased to a peak around $3 \mathrm{kHz}$ and then declined. Above $\sim 5 \mathrm{kHz}$, the level pattern became complex with a series of peaks and valleys. This is in contrast to the ball and rigid sphere model where the spectra were simple and nearly flat (Fig. 2). This difference must be due to in-phase and out-ofphase summations of multiple signal components created by the complex geometry of rabbit's outer ear, head, and body. The rabbit ears also showed considerable gains (e.g., $4 \mathrm{~dB}$ at $0.2 \mathrm{kHz}, 20 \mathrm{~dB}$ at $3 \mathrm{kHz}$ for $90^{\circ}, 10 \mathrm{~cm}$, Figure $5 \mathrm{~d}$ ), whereas the ball and sphere model showed gains near $0 \mathrm{~dB}$ at all frequencies (e.g., $90^{\circ}, 10 \mathrm{~cm}$, Fig. 2A, D).

Across distance, the level in both ears increased with decreasing distance as expected, except at high frequencies (Fig. 5, top and middle rows). The level in the right ear (ear closer to the sound source, top row) generally increased as the sound source azimuth moved from $0^{\circ}$ (directly in front of the rabbit) to $90^{\circ}$. The level in the left ear (middle row) displayed an opposite pattern to the right ear in that the level decreased as the sound source azimuth moved from $0^{\circ}$ to $90^{\circ}$. These opposite changes in the levels of the two ears indicate that the ILD should generally 


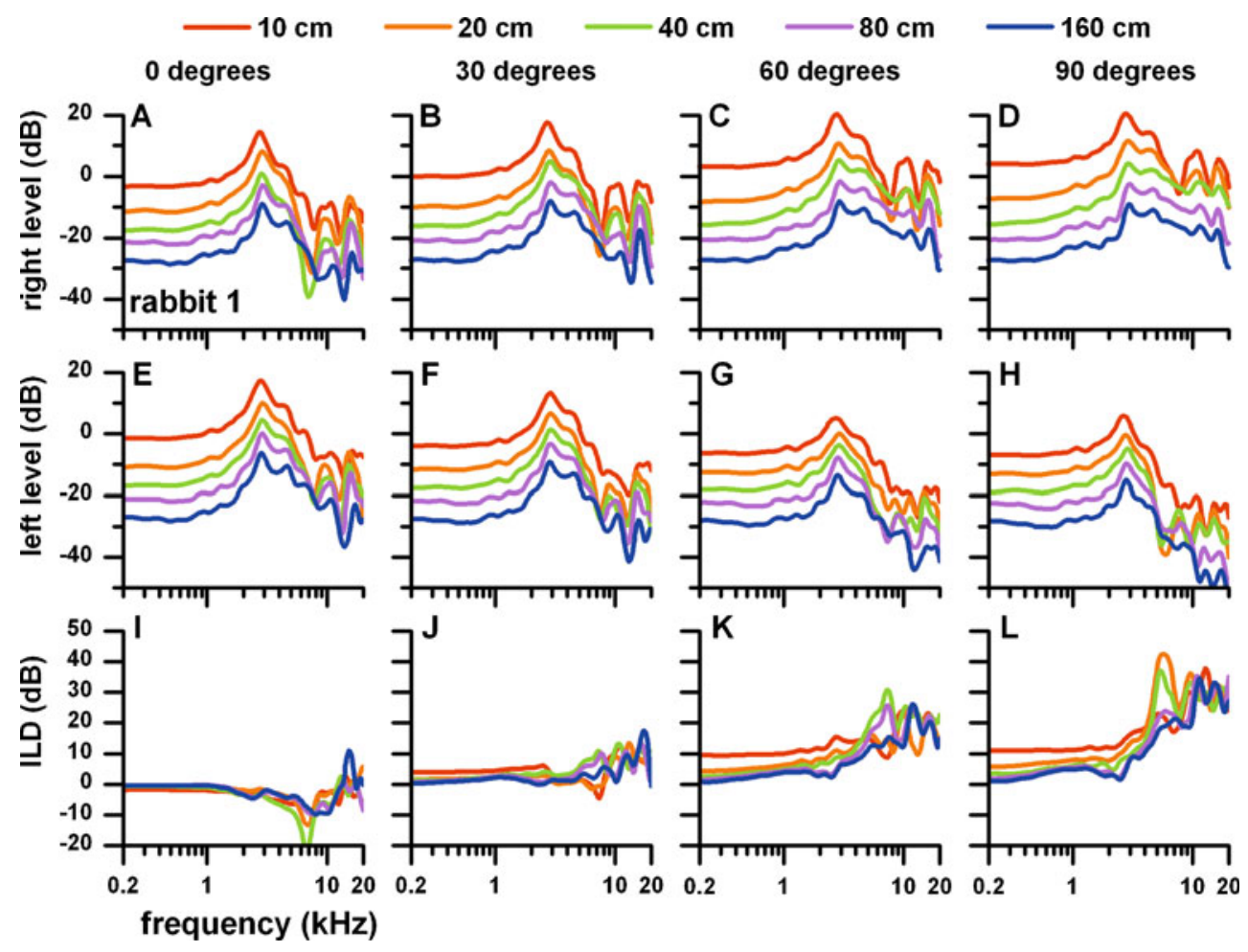

FIG. 5. Monaural levels (top two rows) and ILDs (bottom row) vs. frequency measured at five distances (10, 20, 40, 80, and 160 cm) and four azimuths $\left(0,30,60\right.$, and $\left.90^{\circ}\right)$ in a rabbit $(\# 1)$ in an anechoic chamber.

increase as the sound source azimuth is moved from $0^{\circ}$ to $90^{\circ}$. This prediction was verified by the ILDs (bottom row) derived from the monaural levels. Between 0.2 and $1 \mathrm{kHz}$, the ILD was flat and increased with increasing azimuth and with decreasing distance. At the closest distance $(10 \mathrm{~cm})$, it reached $\sim 10 \mathrm{~dB}$ whereas it was near $0 \mathrm{~dB}$ at the farthest distance $(160 \mathrm{~cm})$. At high frequencies, the ILDs were larger but less systematically related to distance. The maximum ILD was much larger $(42 \mathrm{~dB})$ for the rabbit than for the ball $(15 \mathrm{~dB})$ or the sphere model $(12 \mathrm{~dB})$. This difference must be due to the complex geometry of rabbit's outer ear, head, and body.

To more easily view changes in the monaural levels of the HRTFs across azimuth, Figure 6 re-plots the HRTFs in Figure 5 separately for distances of 10, 40, and $160 \mathrm{~cm}$ at azimuths of $0^{\circ}, 30^{\circ}, 60^{\circ}$, and $90^{\circ}$. For the ear on the side of the sound source (left column, right ear), the spectral peaks and notches became more prominent with decreasing distance for a constant azimuth. For a constant distance, the spectral peaks and notches became more prominent as the azimuth shifted from $90^{\circ}$ to $0^{\circ}$, particularly at further distances (40 and $160 \mathrm{~cm}$ ). For the ear on the opposite side of the sound source (right column, left ear), the trends observed in the right ear are not so clear.

Figure 7 provides a detailed view of how ILD changes across azimuth for four frequency bands (low, 0.2-2; mid, 2-5; high, 5-20; and full, $0.2-20 \mathrm{kHz})$ at five distances $(10,20,40,80$, and $160 \mathrm{~cm})$ for two rabbits, the racquetball, and sphere model. In all cases, the changes of ILD across azimuth were approximately sinusoidal with the maxima and minima near $\pm 90^{\circ}$. Furthermore, the ILDs increased from the low to the high frequency bands with the full-band ILDs being intermediate. However, ILDs in the rabbits were larger than those in the ball or sphere model, particularly for those in the high frequency band. A prominent notch at $\pm 90^{\circ}$ was seen in the ball and model in the high frequency band. This notch is created when the wavelength of the sound is much less than the circumference of a spherical object because the sound waves reaching the opposite side are added in-phase (Duda and Martens 1998). They are absent in the rabbit because the head and pinna are not a spherical object. In general, ILD increased with decreasing distance.

The above relationship is shown in Figure 8 where ILD is plotted as a function of distance for $0^{\circ}, 45^{\circ}$, and $90^{\circ}$ azimuths. In all cases, at $0^{\circ}$ azimuth, ILD hardly changed with distance. At non-zero azimuths, ILD increased monotonically with decreasing distance for the low and mid-frequency bands. In the high frequency band, in the rabbit, there was no systematic relation between ILD and distance. This is related to the complex spectra seen at high frequencies only in the rabbit (Figs. 5 and 6). In contrast, for the ball and model, the systematic relationship between ILD and 

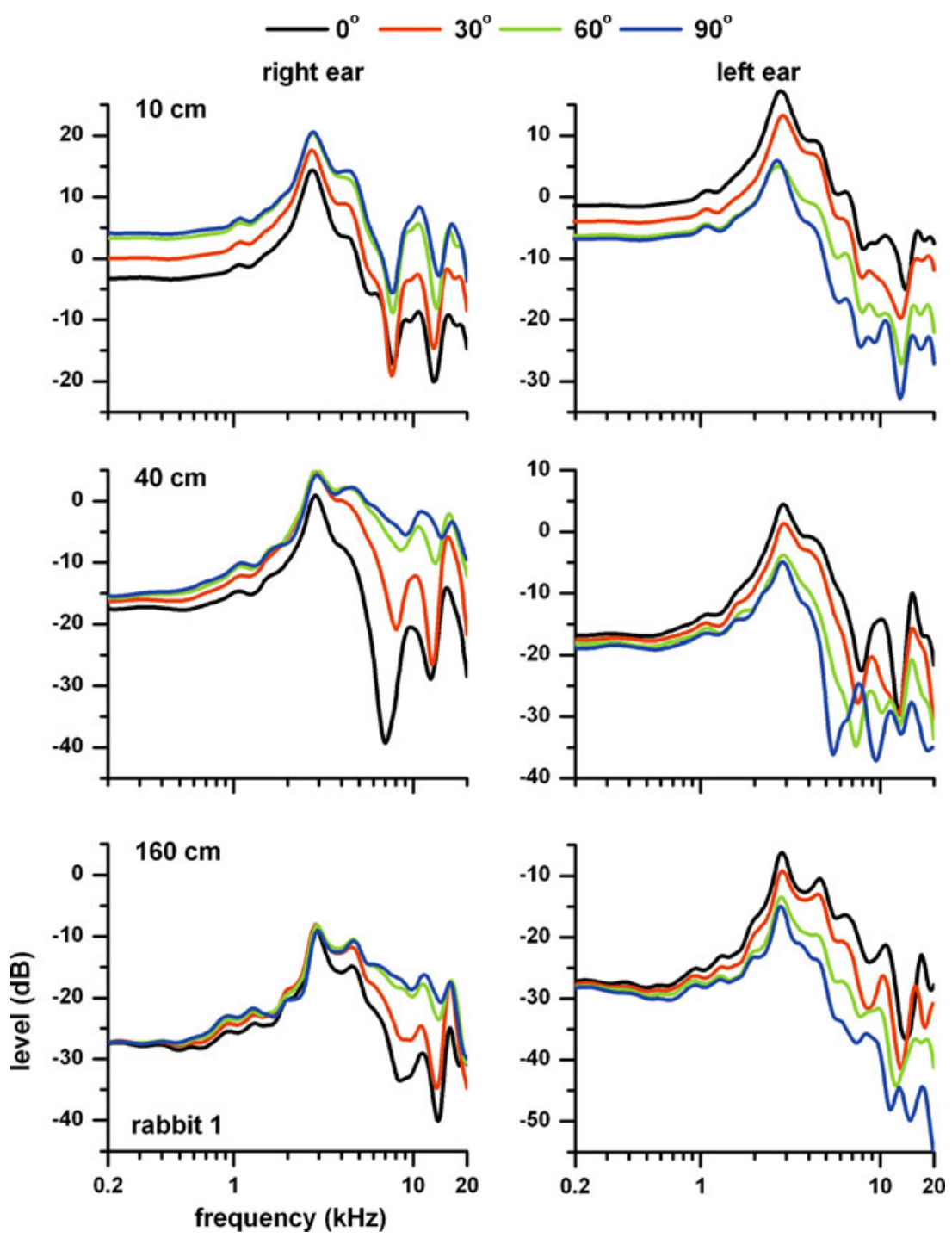

FIG. 6. Re-plot of the information in Figure 10. Each panel represents monaural levels measured at four azimuths $(0,30$, 60 and $\left.90^{\circ}\right)$ at a particular distance (10, 40 , or $160 \mathrm{~cm})$. Left column represents the right ear (on the side of the sound source). Right column represents the left ear (on the opposite side of the sound source). distance was present, even in the high frequency band. This is related to the simple spectra seen at all frequencies in the ball and model (Fig. 2). Another difference is that the ILDs at $45^{\circ}$ were larger than those at $90^{\circ}$ in the ball/sphere model, especially in the high frequency band, whereas ILDs at $90^{\circ}$ were the largest in the rabbit. This is due to the in-phase wave addition for high frequencies in a spherical object (see above).

Figure 9 provides another view, in the form of color contour plots, of the effect of sound source distance, azimuth, and frequency on ILD in the two rabbits. Figure 9A, C plots the ILD as a function of distance and frequency for a sound source at $90^{\circ}$ azimuth. At frequencies below $\sim 3 \mathrm{kHz}$, the ILD albeit small, increased with decreasing distance (A and C). Above $\sim 4 \mathrm{kHz}$, ILDs were much larger and exhibited complex patterns across distance and frequency. While the patterns were similar at lower frequencies $(<3 \mathrm{kHz})$ in the two rabbits, the banded patterns at high frequencies $(>4 \mathrm{kHz})$ were unique to each rabbit. Figure 9B, D plots the ILD as a function of azimuth and frequency for a sound source at $40 \mathrm{~cm}$. At frequencies below $\sim 3 \mathrm{kHz}$, the ILD, albeit small, increased as the azimuth increased from $0^{\circ}$ to $90^{\circ}(\mathrm{B}$, D). Above $\sim 4 \mathrm{kHz}$, ILDs were much larger and exhibited complex patterns across azimuth and frequency. In both rabbits, there was a front-back asymmetry $\left(<90^{\circ}\right.$ vs $\left.>90^{\circ}\right)$, albeit more pronounced in rabbit 2. Again, the banded, high frequency patterns were unique to each rabbit.

Monaural and binaural temporal characteristics of the rabbit

As in the racquetball (Fig. 3), the phase delays in the rabbit were derived as a function of frequency, in the range from 0.2 to $2 \mathrm{kHz}$, for each ear at each sound source location. Figure $10 \mathrm{~A}-\mathrm{E}$ displays the phase delays recorded at each ear for a sound source at 


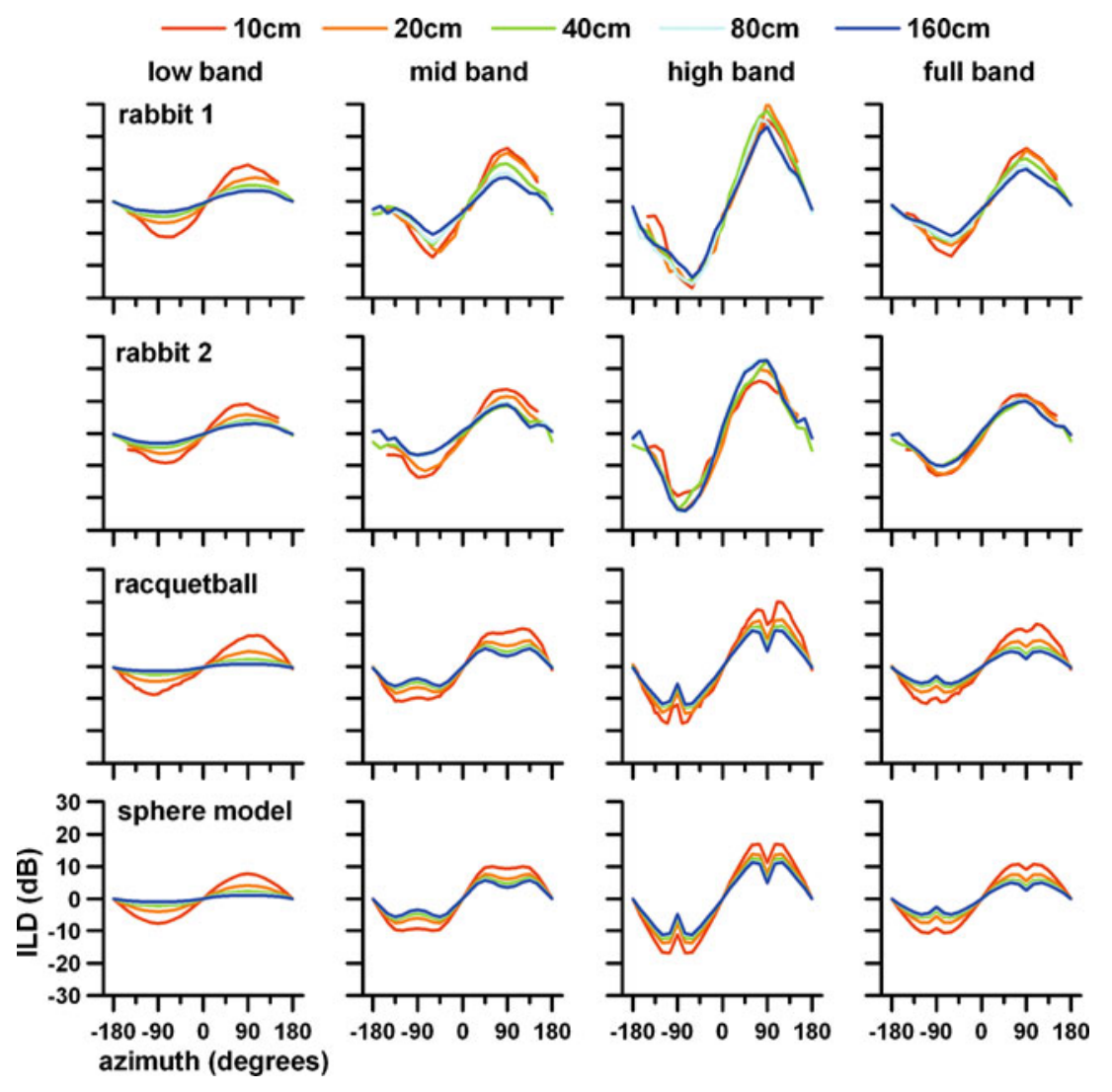

FIG. 7. Low, mid, high, and full-band ILDs vs. azimuth at five distances $(10,20$, 40, 80, and $160 \mathrm{~cm}$ ), for rabbits 1 and 2, a racquetball, and the sphere model.

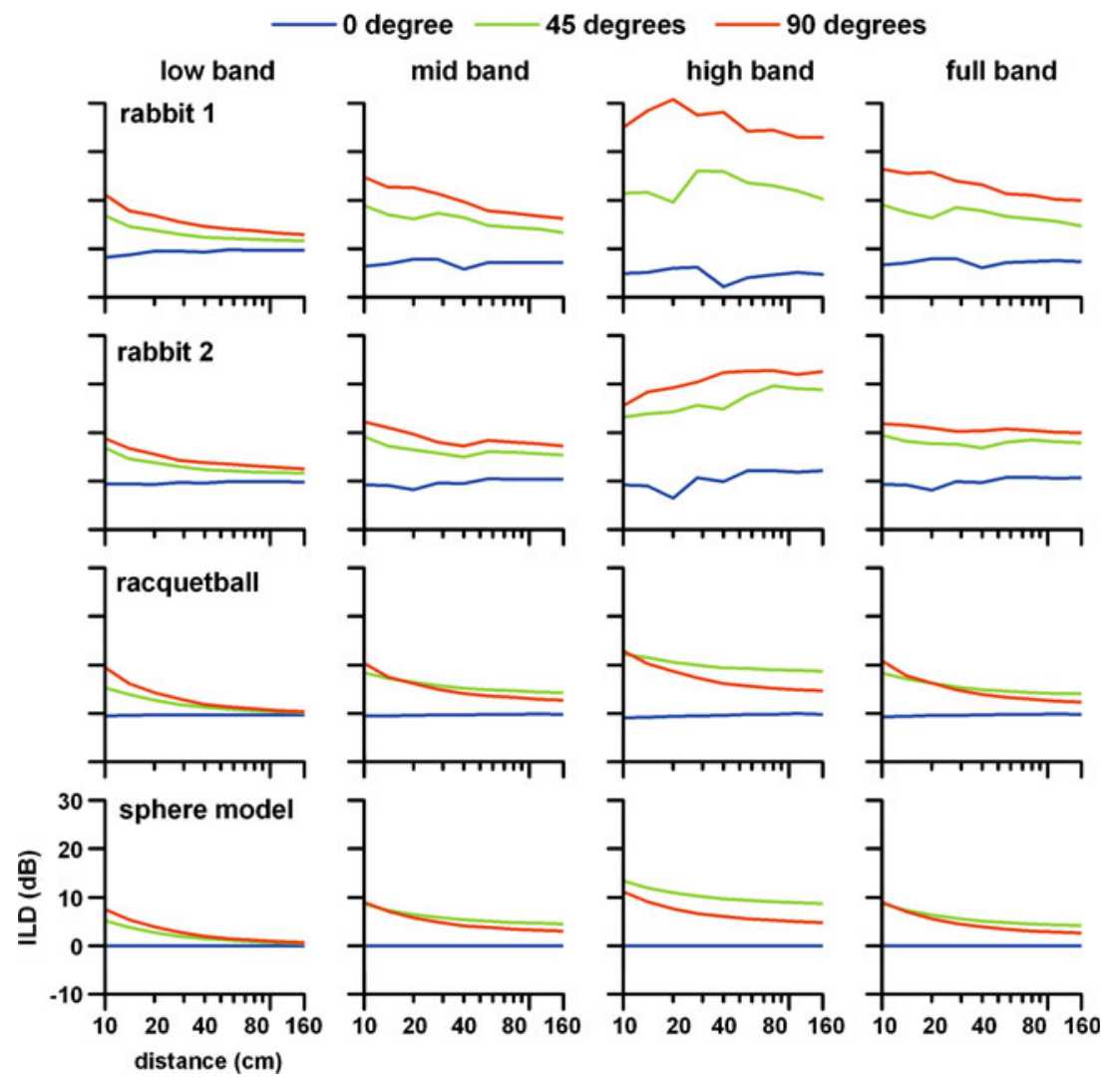

FIG. 8. Re-plot of Figure 7 to show ILD vs. distance at three azimuths $(0,45$, and $\left.90^{\circ}\right)$. 

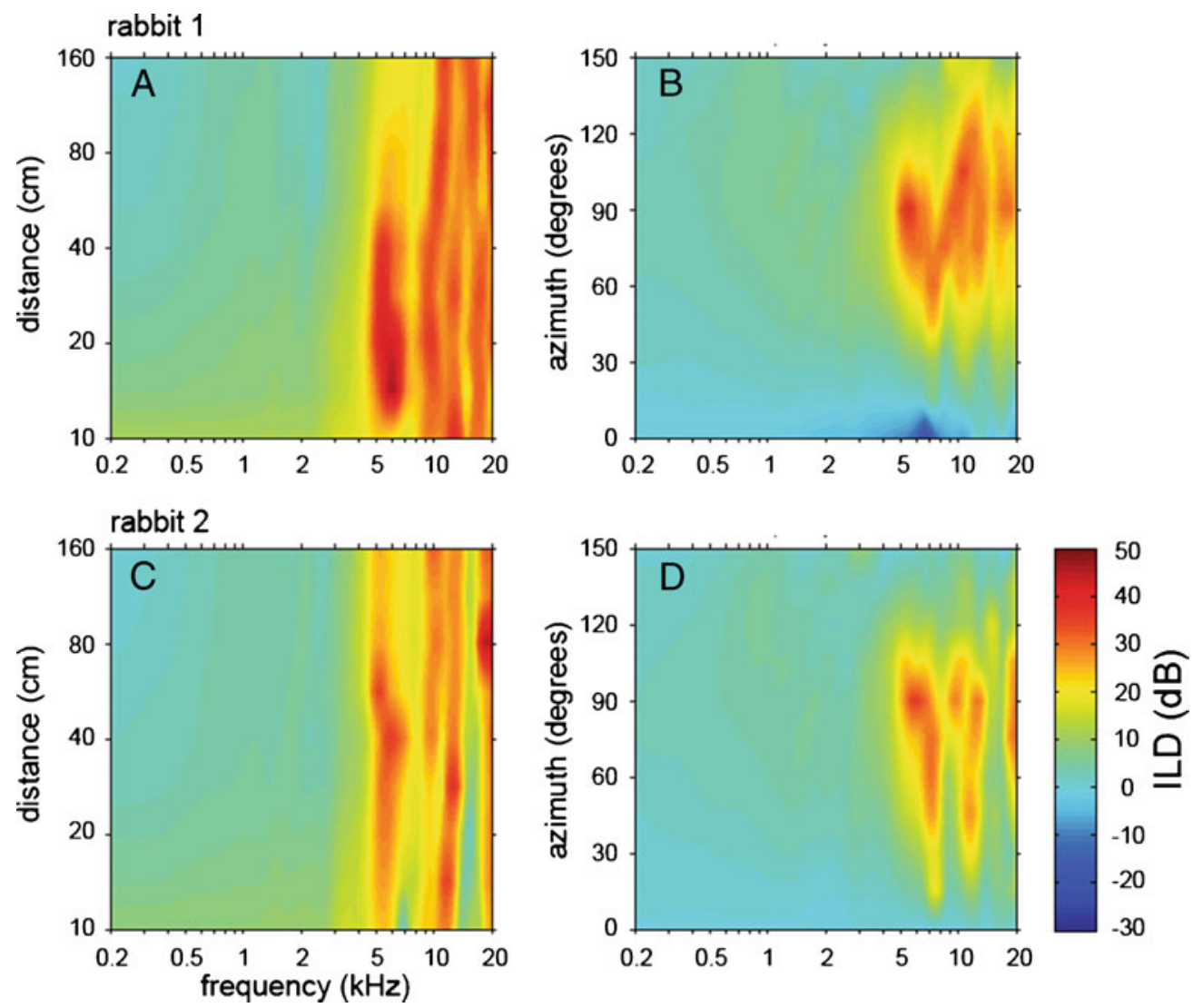

FIG. 9. Color contour plots of ILD as a function of distance and frequency at $90^{\circ}$ for the two rabbits (A, C), and as a function of azimuth and frequency at $40 \mathrm{~cm}$ for the two rabbits (B, D).

$90^{\circ}$ azimuths for distances between 10 and $160 \mathrm{~cm}$ for rabbit 1 . The patterns of monaural delay seen in this rabbit are remarkably similar to those seen in the racquetball. Both measurements show that the delay to the left ear was longer than that to the right ear; the delay to both ears increased with increasing distance; the delay decreased with decreasing frequency, and the magnitude of this decrease increased with increasing distance. In the bottom panel (Fig. 10F) are the ITDs derived from the monaural delays shown above. Between 1.2 and $2 \mathrm{kHz}$, the ITDs were approximately constant across distance. Between 0.2 and $1.2 \mathrm{kHz}$, the ITD changed with frequency and distance. At distances $\geq 80 \mathrm{~cm}$, the ITD generally increased with decreasing frequency, whereas at $10 \mathrm{~cm}$ the ITD decreased with decreasing frequency. At intermediate distances $(20-40 \mathrm{~cm})$, the ITDs fell systematically between these extremes. At $0.2 \mathrm{kHz}$, the ITD decreased from $350 \mu \mathrm{s}$ at $160 \mathrm{~cm}$ to $185 \mu \mathrm{s}$ at $10 \mathrm{~cm}$, a difference of $165 \mu \mathrm{s}(90 \%$ relative to the ITD at $10 \mathrm{~cm}$ ). This difference is much larger than that seen in the racquetball (36 $\mu$ s, 17\%; see Fig. 3). Furthermore, the pattern of ITD across frequency and distance was also different from that of the racquetball (see Fig. 3).

Figure 11 illustrates the relation between ITD and frequency for different azimuths and distances measured in rabbit 1 . At $0^{\circ}$, the functions for various distances superimposed near $0 \mu$ s ITD and were nearly constant across frequency. As the azimuth increased, the ITDs as expected increased, and both the distance- and frequency-dependent changes in ITD became more salient.

Figure 12 displays the ITD as a function of azimuth for different distances in both rabbits, racquetball, and sphere model. Here, the ITDs were obtained by averaging over the low frequency band ( 0.2 to $2 \mathrm{kHz}$ ). In all cases, ITDs changed nearly sinusoidally with azimuth reaching peaks in absolute magnitude at $\pm 90^{\circ}$ azimuth. In the rabbits, the ITDs decreased with decreasing distance whereas distance-dependent change of ITD was much smaller in the ball, and no such change was present in the model.

Figure 13 provides another view, in the form of color contour plots, of the effect of sound source distance, azimuth, and frequency on ITD in the two rabbits. Figure 13A, C plot the ITD as a function of distance and frequency for a sound source at $90^{\circ}$ azimuth. In both rabbits, at frequencies below $\sim 1 \mathrm{kHz}$, the ITD decreased with decreasing distance $(\mathrm{A}, \mathrm{C})$, whereas for frequencies between 1 and $2 \mathrm{kHz}$, ITDs changed minimally with distance. At distances $>20 \mathrm{~cm}$, the ITD increased with decreasing 


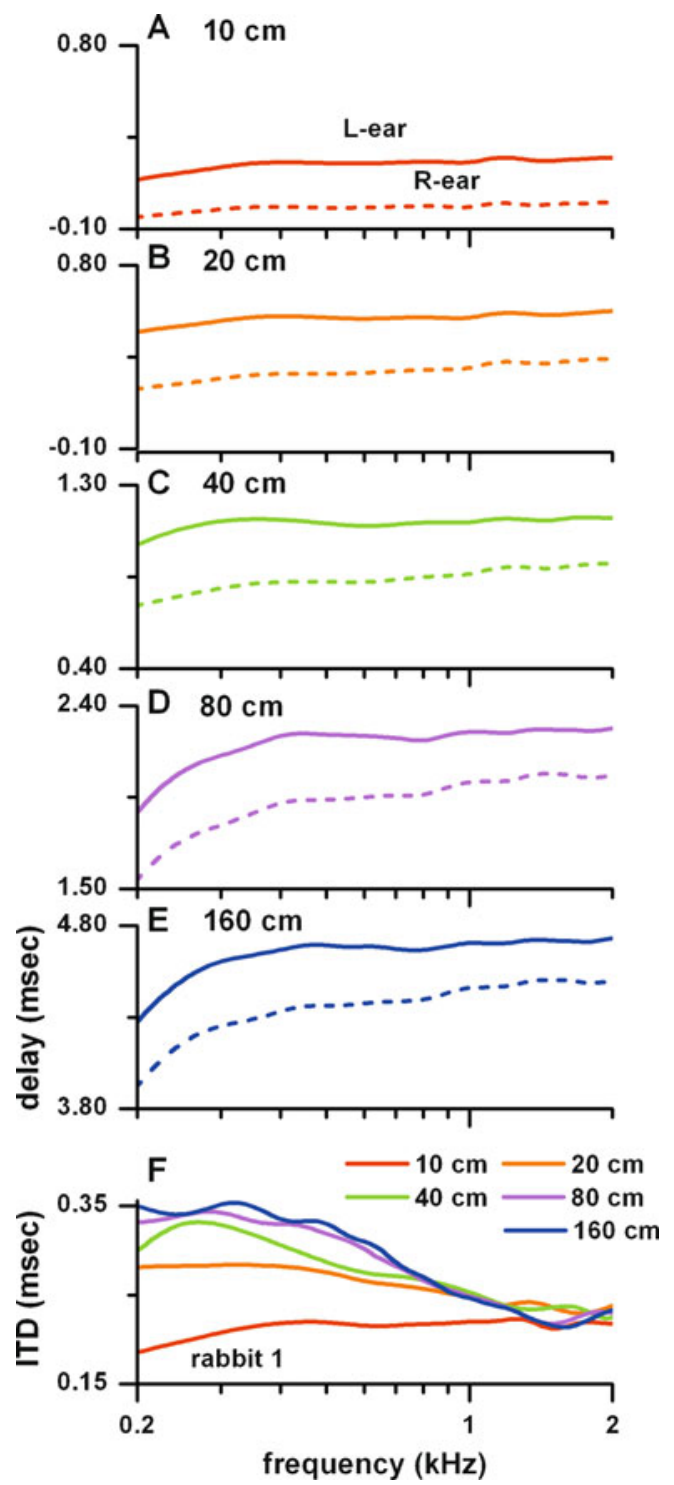

FIG. 10. Monaural delays (top five panels) and ITD (bottom panel) vs. frequency measured at five distances $(10-160 \mathrm{~cm})$ and $90^{\circ}$ azimuth in rabbit 1 .

frequency. The ITD patterns were similar for the two rabbits. Figure 13B, D plot the ITD as a function of azimuth and frequency for a sound source at $40 \mathrm{~cm}$. In both rabbits, the ITD increased with increasing azimuth, reaching a maximum near $90^{\circ}$ (B, D) and tended to increase with decreasing frequency. In both rabbits, there was a front-back asymmetry $\left(<90^{\circ}\right.$ vs. $\left.>90^{\circ}\right)$, albeit more pronounced in rabbit 1 .

\section{Effect of pinna position on ILD and ITD}

All of the previous results from the rabbits were from ear canal measurements where the ears were upright and symmetrical. Here, we show the effects of ear position on ITD and ILD. By rotating the trapeze the right ear could be placed forward, and the left ear placed backward relative to the symmetrical position. The other variant was to reverse this configuration, i.e., right ear backward and left ear forward.

Figure 14A-C shows, for different frequency bands, that when the right ear was forward and left ear backward (red), the ILD function shifted to the left (towards negative azimuths). The opposite shift occurred when the ears were placed in the opposite direction (blue). Although this shift occurred in all frequency bands, the magnitude of these shifts increased from the low $(0.2-2 \mathrm{kHz})$ to the high frequency $(5-20 \mathrm{kHz})$ band. As expected (Fig. 7), the ILD also increased from the low to high frequency band. Relative to the symmetrical ear position, the amount of shift was always larger when the right ear was forward than when the left ear was forward. The asymmetrical shifts may reflect inaccuracies in positioning the ears and/or anatomical asymmetries between the ears. The asymmetrical ear positions also reduced the range of ILDs in all frequency bands. For example, in the high

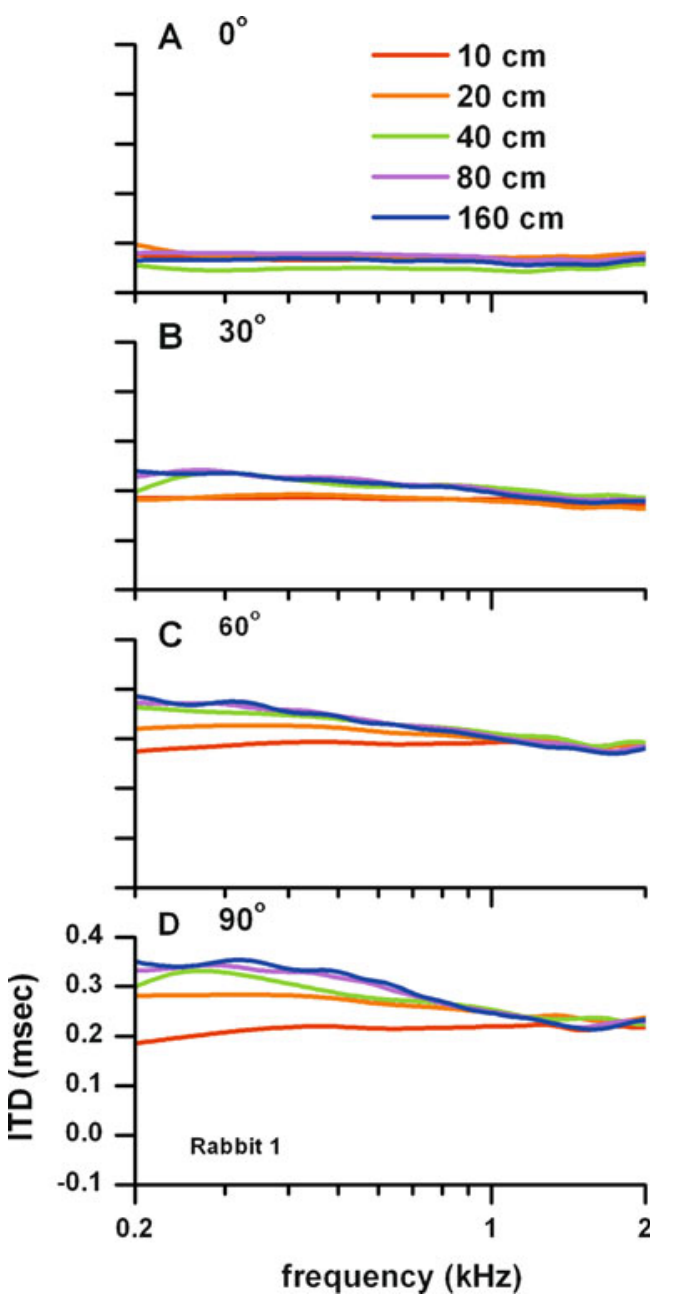

FIG. 11. ITD vs. frequency measured at five distances $(10-160 \mathrm{~cm})$ and four azimuths $\left(0\right.$ to $\left.90^{\circ}\right)$ in rabbit 1 . 


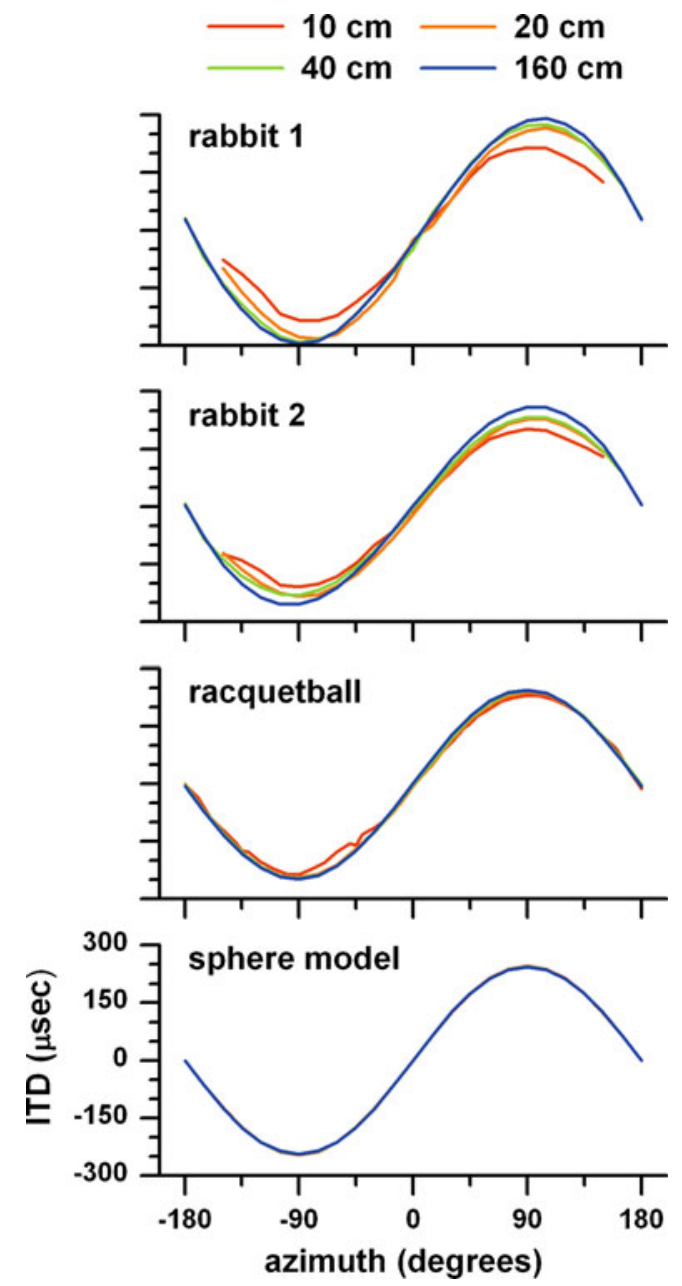

FIG. 12. ITD as a function of azimuth for different distances in rabbits 1 and 2, racquetball, and sphere model. Here, the ITDs were obtained by averaging over the low frequency band $(0.2$ to $2 \mathrm{kHz}$ ). This band averaged ITD was essentially the same as the ITD obtained by cross-correlating the low-frequency-band HRIRs of the two ears.

frequency band (Fig. 14C), the reduction was from $\pm 25.6 \mathrm{~dB}$ (black) to $\pm 18.9 \mathrm{~dB}$ (red) or to $\pm 24.1 \mathrm{~dB}$ (blue) for the opposite ear position. This reduction is presumably because the side-to-side sound path became less obstructed when the ears were asymmetrically positioned.

The shifts in the ITD functions (Fig. 14D) were similar to those seen in the ILD functions (Fig. 14A-C). Relative to the symmetrical ear position the amount of shift was larger when the right ear was forward than when the left ear was forward. The asymmetrical ear positions also reduced the range of ITDs from $\pm 296 \mu$ s to $\pm 259 \mu \mathrm{s}$ (red) or to $\pm 270 \mu$ s (blue) for the opposite ear position. The asymmetrical shifts and reduction in range may reflect the same factors discussed above.

The effects of different outer ear positions on ILD (top panels) and ITD (bottom panels) as a function of frequency and azimuth are shown in the form of color contour plots in Figure 15. As before, ILDs were prominent at frequencies $>\sim 4 \mathrm{kHz}$. As the left ear was moved from a forward (A) to a backward position (C) with the right ear moving in the opposite direction, the center of the ILD patterns shifted from a positive (right side) to a negative (left side) azimuth. As before (Fig. 10), ITD increased with decreasing frequency. With different pinna positions, the ITD pattern shifted in azimuth in an analogous way to the shift in ILD described above.

\section{DISCUSSION}

This is the first study of HRTFs in the rabbit. Our main finding is that features of the rabbit HRTF changed substantially with sound source distance and azimuth. We found that (1) the spectral shape of the HRTF in each ear changed with sound source location; (2) ILD increased with decreasing distance and with increasing frequency. Furthermore, ILDs can be substantial even at low frequencies at close distances; and (3) ITD decreased with decreasing distance and generally increased with decreasing frequency. The information described in this paper extends the literature for acoustic cues for sound localization with respect to distance. The present results will be useful, e.g., for generating virtual auditory space stimuli that can facilitate behavioral and neural studies of sound localization (Kuwada et al. 2010b).

Studies that measured HRTFs as a function of distance are few. Brungart and Rabinowitz (1999) measured sound source distance effects using the human mannequin, KEMAR. Zahorik (2002) and Kuwada et al. (2010a) performed these measurements in humans, and Kim et al. (2008) measured this in barn owls. A rigid sphere model by Duda and Martens (1998) provides a most useful theoretical tool for computing HRTFs for any sound source location in 3-dimensional space.

Effects of distance and azimuth on spectral shape of HRTFs

Distance-dependent changes in spectral shape occurred mainly at frequencies above $\sim 3 \mathrm{kHz}$ in the rabbit and in the studies cited above. This change was not observed in the racquetball or spherical model, implying that these spectral changes arise from the phase cancelations created by the complex shapes of the head and outer ear. This is consistent with the finding that pinna removal in the rat and the cat led to a reduction of the gain and smoothing of the 

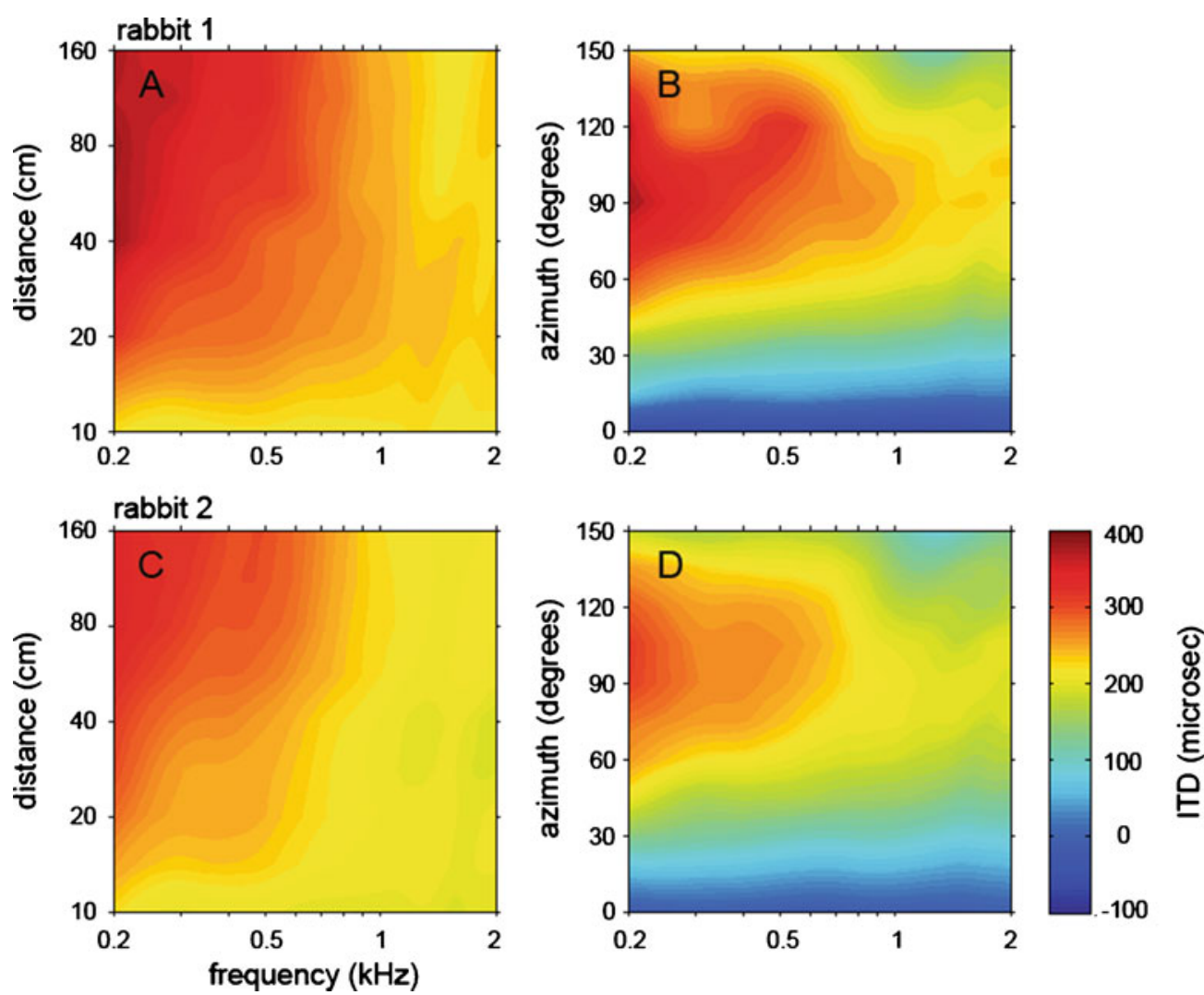

FIG. 13. Color contour plot of ITD as a function of distance and frequency at $90^{\circ}$ for the two rabbits (A, C) and as a function of azimuth and frequency at $40 \mathrm{~cm}$ for the two rabbits (B, D).

spectral shape (Wiener et al. 1966; Koka et al. 2008; Tollin and Koka 2009).

Both the rabbit and human exhibited remarkably similar positive gains. For example, at a distance of $14 \mathrm{~cm}$ and $90^{\circ}$ azimuth, the maximum gain was $15.3 \mathrm{~dB}$ at $2.8 \mathrm{kHz}$ in the rabbit (data not shown) and $14.6 \mathrm{~dB}$ at $4.3 \mathrm{kHz}$ in the human (Kuwada et al. 2010a, b). This similarity was also observed when cats were compared with humans regarding both the maximum gain and the frequency at the maximum gain (Wiener and Ross 1946; Wiener et al. 1966).

We showed that the pattern of change of the spectral shape across distance and azimuth was recognizable in the ear on the same side as the sound source. In contrast, the changes of the spectral shape in the opposite ear were more complex (Fig. 6). This is consistent with observation that human monaural sound localization is based mainly on the information in the ear on the side of sound source (Humanski and Butler 1988; Morimoto 2001; Jin et al. 2004; Van Wanrooij and Van Opstal 2004). However, Van Wanrooij and Van Opstal (2004) observed that the head shadowing effect was also a contributing factor to monaural sound localization, thus implicating a role of monaural information in the ear opposite to the sound source. Slattery and Middlebrooks (1994) observed that some unilaterally deaf humans were able to achieve reasonably accurate sound localization whether the sound source was on the same side or opposite side of the intact ear. This further implies that the information from a sound source through the intact ear either on the same side or the opposite side contributes to sound localization.

Effects of distance, azimuth, and frequency on ILD

In the rabbit, when the sound source was off the median plane, the ILD increased with decreasing sound source distance. At close distances, ILDs could be substantial even at low frequencies. Brungart (1999) showed in humans that very low-frequency ILDs are important for distance perception. The increase in ILD with decreasing distance has been reported in humans, a human mannequin, and barn owls cited above. The largest ILDs were seen at lateral sound source positions and at high frequencies. This has been extensively reported in humans (Wightman and Kistler 1998) and different nonhuman species (cat: Musicant et al. 1990; gerbil: Maki and Furukawa 2005; ferret: Schnupp et al. 2003; guinea pig: Sterbing et al. 2003; rat: Koka et al. 2008; barn owl: Keller et al. 1998). 

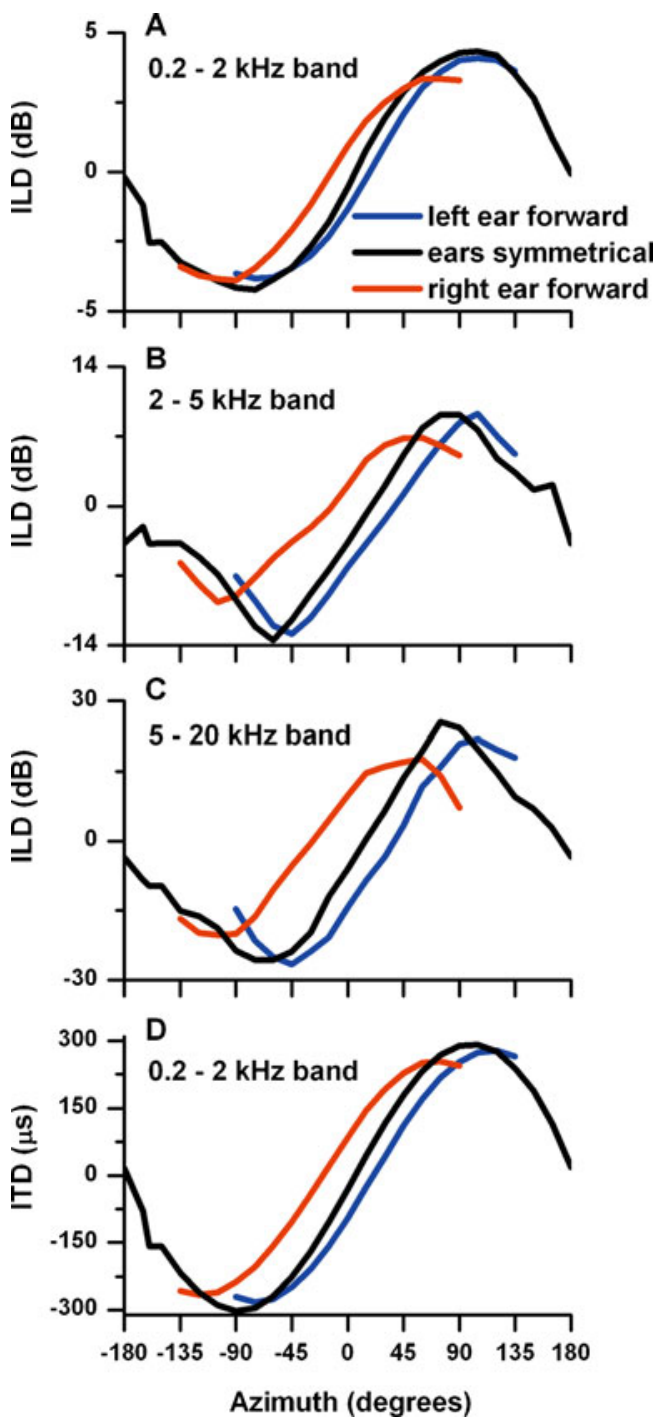

FIG. 14. Effect of pinna position on ILD and ITD in rabbit 1 for a sound source at a distance of $56 \mathrm{~cm}$. A Low-frequency band averaged ILD $(0.2-2 \mathrm{kHz})$ vs. azimuth at three different positions of the pinnae: symmetrical position (black line), right ear forward and left ear backward (red line), and when the left ear forward and the right ear backward (blue line). B Same as A, except that ILDs were averaged over the mid-frequency band $(2-5 \mathrm{kHz})$. C Same as $\mathbf{A}$, except that ILDs were averaged over the high frequency band (5-20 kHz). D ITD averaged over the low frequency band at the same three ear positions described in $\mathbf{A}$.

ILDs in the rabbit were much larger than those in the racquetball and sphere model. This difference must be due to the complex geometry of the rabbit's outer ear, head, and body. Consistent with this view is the finding that removal of the pinna in the rat reduced the ILDs (Koka et al. 2008).

Effects of distance, azimuth, and frequency on ITD

The ITD behavior in the rabbit across distance and frequency was partly similar and partly different than that in the racquetball. Both showed a decrease in ITD with decreasing distance, albeit much larger, by a factor of 4.4 in the rabbit. The maximum ITD was greater in the rabbit ( 350 vs. $250 \mu \mathrm{s}$ ) which may arise from the rabbit's outer ears and body. This may be consistent with the finding of Koka et al. (2008) that pinna removal reduced the envelope ITDs in the rat. At frequencies $<1 \mathrm{kHz}$ and distances $\geq 40 \mathrm{~cm}$, the ITD in the rabbit increased with decreasing frequency, whereas, in the ball, it was approximately constant. This constant ITD across frequency of the ball was well-explained by the rigid sphere model.

The increase in ITD with decreasing frequency at far distances described in the rabbit has also been reported in the human mannequin (Kuhn 1977; Brungart and Rabinowitz 1999), cat (Roth et al. 1980), and guinea pig (Sterbing et al. 2003). These observations refute the common misconception that the physiological range of ITD is constant across frequency. The observation that the best ITD of a neuron increases with decreasing best frequency (McAlpine et al. 2001) may be, at least in part, due to this acoustic phenomenon.

The effect of distance and frequency on ITD has been studied in the human mannequin and the matching rigid sphere model by Brungart and Rabinowitz (1999). They found that ITD increased with decreasing frequency in both the human mannequin and the sphere model. This is consistent with the present finding and in a separate measurement of the human mannequin as reported by Kuhn (1977). Furthermore, Brungart and Rabinowitz (1999) found that for frequencies above $0.5 \mathrm{kHz}$, ITD increased with decreasing distance in both the mannequin and sphere model, albeit smaller in the sphere model. Our findings of a decrease in ITD with decreasing distance in the rabbit and the racquetball are seemingly contradictory to those of Brungart and Rabinowitz (1999). However, Kuwada et al. (2010a) found that, in humans and in a human-head-size ball, ITD did decrease with decreasing distance at frequencies between 0.2 and $\sim 0.5 \mathrm{kHz}$, a pattern much like that seen in the rabbit. Since Brungart and Rabinowitz (1999) did not include ITDs for frequencies below $0.5 \mathrm{kHz}$, the above pattern was missed.

The behaviors of different diameter rigid sphere models are identical when the variables are properly normalized (Duda and Martens 1998). In this approach, the normalized frequency is proportional to the diameter of the sphere. Considering that we used a diameter of $0.056 \mathrm{~m}$ for the rabbit-size sphere and Brungart and Rabinowitz (1999) used a diameter of $0.18 \mathrm{~m}$ for the human-size sphere, the normalized frequency is 3.21 times higher for the human-size 

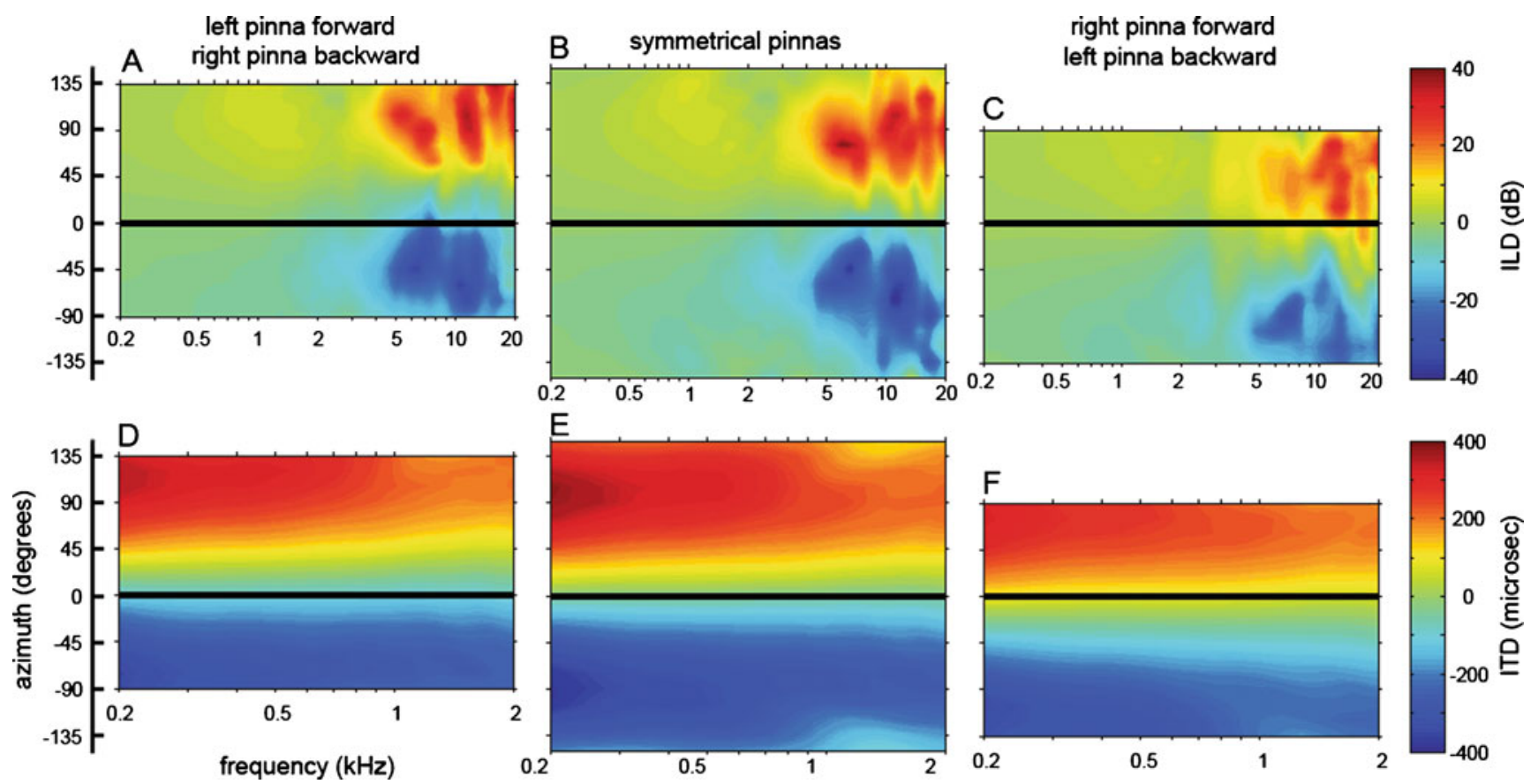

FIG. 15. Color contour plot of ILD and ITD representing the information in Figure 14. A-C: ILD as a function of azimuth and frequency for the three pinna positions. D-F: ITD as a function of azimuth and frequency for the three pinna positions.

sphere. For example, $2 \mathrm{kHz}$ for the rabbit-size sphere is equivalent to $0.62 \mathrm{kHz}$ for the human-size sphere. At these frequencies, ITD increased with decreasing distance in both size spheres.

The acoustic data indicate that the maximum ITDs were 301 and $254 \mu$ s for rabbit 1 and 2, respectively. The bulk of the characteristic delays (CD) and best ITDs of neurons in the dorsal nucleus of the lateral lemniscus (DNLL) and inferior colliculus (IC) recorded in the unanesthetized rabbit were within $\pm 300 \mu$ s (DNLL, $C D=92 \%$, best $\mathrm{ITD}=88 \%, \mathrm{IC}, \mathrm{CD}=83 \%$, best $\mathrm{ITD}=79 \%$; Kuwada et al. 2006) and heavily biased towards the contralateral azimuthal field. Thus, the bulk of the neural CDs and best ITDs fall within the range of acoustic ITDs measured in the rabbit.

\section{Resolving ambiguity in distance and azimuth}

Different combinations of sound source azimuth and distance can produce similar values of an acoustic cue, thus creating ambiguity in localizing a sound source. Is it possible to resolve such ambiguities by unique combinations of multiple acoustic cues? For example, ITD alone is an ambiguous indicator of azimuth because it changes with distance. This is illustrated in Figure 8 for rabbit 1 . The ITD of $-234 \mu \mathrm{s}$ corresponds to a source either at $10 \mathrm{~cm},-90^{\circ}$ or at $40 \mathrm{~cm},-45^{\circ}$. This ambiguity can be resolved if ITD and ILD cues are combined as follows. In Figure 4, for the same rabbit, the low band ILD is $11 \mathrm{~dB}$ at $10 \mathrm{~cm}, 90^{\circ}$ or $2.3 \mathrm{~dB}$ at $40 \mathrm{~cm}, 45^{\circ}$. So, when the two cues are combined, the ambiguity is resolved, e.g., a combination of $-234 \mu \mathrm{s}$ ITD and $11 \mathrm{~dB}$ ILD uniquely identifies the location to be at a distance of $10 \mathrm{~cm}$ and an azimuth of $-90^{\circ}$. In general, combining multiple acoustic cues derived from monaural and binaural spectra would help resolve ambiguities in sound source location (Slattery and Middlebrooks 1994; Jin et al. 2004; Van Wanrooij and Van Opstal 2004).

\section{Effects of pinna position on binaural cues}

The position of the pinna could markedly alter the ILD and ITD cues for a fixed sound source location. Thus, given its mobile pinna, the rabbit could use these ear movements to place the sound source location at the center of the ILD-ITD ranges. Furthermore, the shifts in ILD and ITD in azimuth were remarkably similar indicating that the information based on these cues was coherent. For a broadband sound source, the high-frequency ILD information and the low-frequency ITD information would lead to a consistent determination of the sound source location. Pinna position was previously found to affect the HRTFs in cats (Young et al. 1996). It has also been shown that neural spatial receptive field in the superior colliculus of the cat shifted in responses to changes in pinna position (Middlebrooks and Knudsen 1987). 


\section{ACKNOWLEDGEMENT}

We thank Dr. Susanne Sterbing-D'Angelo and Mr. Jason Sijie Wang for their assistance. We also thank Drs. Anthony Brammer and Pavel Zahorik for their advice in the design of our sound source. This study was supported by NIH grant R01 DC002178.

\section{OPEN ACCESS}

This article is distributed under the terms of the Creative Commons Attribution Noncommercial License which permits any noncommercial use, distribution, and reproduction in any medium, provided the original author(s) and source are credited.

\section{REFERENCES}

Blauert J (1997) Spatial Hearing: The Psychophysics of Human Sound Localization, revised edition. Cambridge, MA: MIT Press

BRUNGaRT DS (1999) Auditory localization of nearby sources. III. Stimulus effects. J Acoust Soc Am 106:3589-3602

BRUngart DS, Rabinowitz WM (1999) Auditory localization of nearby sources. Head-related transfer functions. J Acoust Soc Am 106:1465-1479

Carney LH, Кoch K, Abrams R, Idrobo F (2010) Acoustic source distance discrimination in rabbit. AssocResOtolarngol Abst 33:313

Coffey CS, Ebert CS Jr, Marshall AF, Skaggs Jd, Falk SE, Crocker WD, Pearson JM, Fitzpatrick DC (2006) Detection of interaural correlation by neurons in the superior olivary complex, inferior colliculus and auditory cortex of the unanesthetized rabbit. Hear Res 221:1-16

Devore S, Delgutte B (2008) Effects of reverberation on neuronal sensitivity to fine time structure and envelope ITD in the inferior colliculus of awake rabbit. Assoc Res Otolarngol Abstr $31: 868$

Duda R, Martens W (1998) Range dependence of the responses of a spherical head model. J Acoust Soc Am 104:3048-3058

Ebert CS Jr, Blanks DA, Patel Mr, Coffey CS, Marshall AF, FitzPATRICK DC (2008) Behavioral sensitivity to interaural time differences in the rabbit. Hear Res 235:134-142

Fitzpatrick DC, Kuwada S, Batra R (2000) Neural sensitivity to interaural time differences: beyond the Jeffress model. J Neurosci 20:1605-1615

Fitzpatrick DC, Roberts JM, Kuwada S, Kim DO, Filipovic B (2009) Processing temporal modulations in binaural and monaural auditory stimuli by neurons in the inferior colliculus and auditory cortex. J Assoc Res Otolaryngol 10:579-593

Heffner H, Masterton B (1980) Hearing in Glires: domestic rabbit, cotton rat, feral house mouse, and kangaroo rat. J Acoust Soc Am 68:1584-1599

Humanski RA, ButLer RA (1988) The contribution of the near and far ear toward localization of sound in the sagittal plane. J Acoust Soc Am 83:2300-2310

Jin C, Corderoy A, Carlile S, van Schaik A (2004) Contrasting monaural and interaural spectral cues for human sound localization. J Acoust Soc Am 115:3124-3141

Keller CH, Hartung K, TAKahashi TT (1998) Head-related transfer functions of the barn owl: measurement and neural responses. Hear Res 118:13-34
Kim DO, Moiseff A, Turner JB, Gull J (2008) Acoustic cues underlying auditory distance in barn owls. Acta Otolaryngol 128:382-387

Koka K, Read HL, Toldin DJ (2008) The acoustical cues to sound location in the rat: measurements of directional transfer functions. J Acoust Soc Am 123:4297-4309

KunN G (1977) Model for the interaural time differences in the azimuthal plane. J Acoust Soc Amer 82:157-167

Kulkarni A, Colburn HS (1998) Role of spectral detail in soundsource localization. Nature 396:747-749

Kuwada S, Fitzpatrick DC, Batra R, Ostapoff EM (2006) Sensitivity to interaural time differences in the dorsal nucleus of the lateral lemniscus of the unanesthetized rabbit: comparison with other structures. J Neurophysiol 95:1309-1322

Kuwada C, Bishop B, Kuwada S, Kim D (2010a) Acoustic recordings in human ear canals to sounds at different locations. Otolaryngol Head Neck Surg 142:615-617

KuWADA S, BISHOP B, Kim D (2010b) Responses of inferior colliculus neurons in the unanesthetized rabbit to virtual auditory space stimuli. AssocResOtolarngol Abst 33:838

MaKi K, FurukaWA S (2005) Acoustical cues for sound localization by the Mongolian gerbil, Meriones unguiculatus. J Acoust Soc Am 118:872-886

McAlpine D, Jiang D, Palmer AR (2001) A neural code for lowfrequency sound localization in mammals. Nat Neurosci 4:396401

Middlebrooks JC, Green DM (1990) Directional dependence of interaural envelope delays. J Acoust Soc Am 87:2149-2162

Middlebrooks JC, Knudsen EI (1987) Changes in external ear position modify the spatial tuning of auditory units in the cat's superior colliculus. J Neurophysiol 57:672-687

Moller H, Sorensen MF, Hammershoi D, Jensen CB (1995) Headrelated transfer functions of human subjects. J Audio Eng Soc 43:300-321

Morimoto M (2001) The contribution of two ears to the perception of vertical angle in sagittal planes. J Acoust Soc Amer 109:15961603

Musicant AD, Chan JC, Hind JE (1990) Direction-dependent spectral properties of cat external ear: new data and crossspecies comparisons. J Acoust Soc Am 87:757-781

Nelson PC, Carney LH (2007) Neural rate and timing cues for detection and discrimination of amplitude-modulated tones in the awake rabbit inferior colliculus. J Neurophysiol 97:522-539

Oppenheim A, Schafer R, Buck J (1999) Discrete-Time Signal Processing. Upper Saddle River, NJ: Prentice Hall

Roth GL, Kochar RK, Hind JE (1980) Interaural time differences: implications regarding the neurophysiology of sound localization. J Acoust Soc Am 68:1643-1651

Schnupp JW, Bоoth J, King AJ (2003) Modeling individual differences in ferret external ear transfer functions. J Acoust Soc Am 113:2021-2030

Slattery WH 3RD, Middlebrooks JC (1994) Monaural sound localization: acute versus chronic unilateral impairment. Hear Res 75:38-46

Stanford TR, KuwAda S, Batra R (1992) A comparison of the interaural time sensitivity of neurons in the inferior colliculus and thalamus of the unanesthetized rabbit. J Neurosci 12:3200-3216

Sterbing SJ, Hartung K, Hoffmann KP (2003) Spatial tuning to virtual sounds in the inferior colliculus of the Guinea pig. J Neurophysiol 90:2648-2659

Toluin D, KоKa K (2009) Postnatal development of sound pressure transformations by the head and pinnae of the cat: monaural characteristics. J Acoust Soc Am 125:980-994

VAn Wanrooij MM, Van Opstal AJ (2004) Contribution of head shadow and pinna cues to chronic monaural sound localization. J Neurosci 24:4163-4171 
Wiener F, Ross D (1946) The pressure distribution in the auditory canal in a progressive sound field. J Acoust Soc Am 18:401-408

Wiener F, Pfeiffer R, Backus AS (1966) On the sound pressure transformation by the head and auditory meatus of the cat. Acta Oto-Laryngologica 61:255-269

Wightman FL, Kistler DJ (1989) Headphone simulation of free-field listening. II: Psychophysical validation. J Acoust Soc Am 85:868-878

Wightman F, Kistler D (1998) Of Vulcan ears, human ears and 'earprints'. Nat Neurosci 1:337-339
Young ED, Rice JJ, Tong SC (1996) Effects of pinna position on head-related transfer function in the cat. J Acoust Soc Am 99:3064-3076

ZAноRIK P (2002) Assessing auditory distance perception using virtual acoustics. J Acoust Soc Am 111:1832-1846

Zheng L, Early SJ, Mason CR, Idrobo F, Harrison JM, Carney LH (2002) Binaural detection with narrowband and wideband reproducible noise maskers: II. Results for rabbit. J Acoust Soc Am 111:346-356 